\title{
SPECTRAL ENERGY DISTRIBUTION AND BOLOMETRIC LUMINOSITY OF THE COOL BROWN DWARF GLIESE 229B
}

\author{
K. Matthews, T. NaKajima, S. R. Kulkarni, And B. R. Oppenheimer \\ Palomar Observatory, 105-24, Caltech, Pasadena, California 91125 \\ Electronic mail: kym@caltech.edu \\ Received 1996 April 18; revised 1996 July 3
}

\section{NASA-CR-204651}

ABSTRACT

Infrared broadband photometry of the cool brown dwarf Gliese 229B extending in wavelength from 0.8 to $10.5 \mu \mathrm{m}$ is reported. These results are derived from both new data and reanalyzed, previously published data. Existing spectral data reported have been rereduced and recalibrated. The close proximity of the bright Gliese $229 \mathrm{~A}$ to the dim Gliese $229 \mathrm{~B}$ required the use of special techniques for the observations and also for the data analysis. We describe these procedures in detail. The observed luminosity between 0.8 and $10.5 \mu \mathrm{m}$ is $(4.9 \pm 0.6) \times 10^{-6} L_{\odot}$. The observed spectral energy distribution is in overall agreement with a dust-free model spectrum by Tsuji et al. for $T_{\text {eff }} \approx 900 \mathrm{~K}$. If this model is used to derive the bolometric correction, the best estimate of the bolometric luminosity is $6.4 \times 10^{-6} L_{\odot}$ and $50 \%$ of this luminosity lies between 1 and $2.5 \mu \mathrm{m}$. Our best estimate of the effective temperature is $900 \mathrm{~K}$. From the observed near-infrared spectrum and the spectral energy distribution, the brightness temperatures $\left(T_{B}\right)$ are estimated. The highest, $T_{B}=1640$ $\mathrm{K}$, is seen at the peak of the $J$ band spectrum, while the lowest, $T_{B} \leqslant 600 \mathrm{~K}$, is at $3.4 \mu \mathrm{m}$, which corresponds to the location of the fundamental methane band. (C) 1996 American Astronomical Society.

\section{INTRODUCTION}

Earlier, we reported the discovery of a cool brown dwarf, Gliese 229B (hereafter Gl 229B). It is assumed to be a companion of the nearby star Gliese 229A (hereafter Gl 229A) because they exhibit common proper motion (Nakajima et al. 1995, N95). In N95, we presented broadband photometry of the brown dwarf between 0.65 and $2.2 \mu \mathrm{m}$ and estimated the bolometric luminosity $\left(L_{\text {bol }}<10^{-5} L_{\odot}\right)$ and the effective temperature $\left(T_{\text {eff }}<1200 \mathrm{~K}\right)$. In a second paper, we presented the near-infrared $(1.0-2.5 \mu \mathrm{m})$ spectrum of $\mathrm{Gl}$ $229 \mathrm{~B}$, and reported the first detection of methane absorption bands (Oppenheimer et al. 1995, O95).

Here we report narrowband imaging photometry of $\mathrm{Gl}$ 229B between 3 and $4 \mu \mathrm{m}$ and broadband aperture photometry of Gl 229A and Gl 229B at $2.2(K), 3.7\left(L^{\prime}\right), 4.8(M)$, and $10.5 \mu \mathrm{m}(N)$. Due to the large flux ratios between, and the small angular separation of, Gl 229A and Gl 229B, considerable effort was made to minimize the influence of the diffracted and scattered light of Gl 229A on the photometry of $\mathrm{Gl} 229 \mathrm{~B}$. We describe the observations and data reduction in Sec. 2 and Sec. 3, respectively. The near-infrared magnitudes $(J, H, K, K \mathrm{~s})$ reported in $\mathrm{N} 95$ were found to be in error because of incorrect accounting for the diffracted light of Gl 229A. The near-infrared, low-resolution spectral data reported in $\mathrm{O} 95$ were also reanalyzed and recalibrated (Sec. 3). In Sec. 4, we discuss the spectral characteristics of the photospheric emission of Gl 229B and estimate the bolometric luminosity with the aid of model spectra by Tsuji et al. (1995, 1996).

\section{OBSERVATIONS}

The journals of observations of Gl 229A and Gl 229B are given in Table 1 . The near-infrared photometry at $Z, J, H$, $K$ s, and $K$ is reported in N95. The near-infrared spectroscopy is reported in O95. The new observations are discussed below. In Sec. 3 we present recalibrated photometry and spectroscopy of the data presented in N95 and O95. These supersede the results reported in N95 and 095 .

\section{$2.13 .8 \mu \mathrm{m}$ Observations}

The observations at $3.8 \mu \mathrm{m}$ were made in photometric conditions on the Hale 200-inch telescope on 1995 October 11 UT using the same equipment (D78, a camera similar to NIRC which is described below) that was used for the observations on 1995 September 14 (N95 and 095). The only difference in the observational technique was that the chopping secondary was used to move the focal plane on the sky and that only the central 8 by 8 arcsec, $(64 \times 64$ pixels) portion of the detector was read out. A single observation consisted of the following steps:

(1) Six 68 millisecond frames were added into the image buffer.

(2) The chopper moved the focal plane 5 arcsec west on the sky and six 68 millisecond frames were added into the sky buffer. The chopper then moved the focal plane 5 arcsec east back to the original position.

(3) Steps 1 and 2 were repeated for 100 cycles.

(4) The telescope was then moved 5 arcsec east, and steps 1,2 , and 3 were repeated with the buffers interchanged.

(5) The final image was formed by subtracting the two 
TABLE 1. (a). Journal of observations and photometry of Gl 229 A.

\begin{tabular}{|c|c|c|c|c|c|c|c|}
\hline $\begin{array}{l}\text { Date } \\
95 \text { UT } \\
\text { (1) }\end{array}$ & $\begin{array}{l}\text { Band } \\
\text { (2) }\end{array}$ & $\begin{array}{c}\lambda \text { range } \\
(\mu \mathrm{m}) \\
(3)\end{array}$ & Telescope & $\begin{array}{c}\text { Instrument } \\
\text { (5) }\end{array}$ & $\begin{array}{l}\text { Mode } \\
(6)\end{array}$ & mag & $\begin{array}{c}\text { flux } \\
\text { (Jy) } \\
\text { (8) } \\
\end{array}$ \\
\hline $23 \mathrm{NC}$ & $\mathrm{K}$ & $2.02-2.43$ & $\mathrm{H} 200$ & $(\mathrm{InSb})$ & Chop & 3.9 & 17.0 \\
\hline & $L^{\prime}$ & & & & & 4.0 & 6.4 \\
\hline & $M$ & & & Plotometer( $(\ln S b)$ & Chop & 4.0 & 3.8 \\
\hline $9 \& 22 \mathrm{NOV}$ & N & $8.0-13.1$ & H200 & Bolometer(Ge) & Chop & 3.9 & 1.02 \\
\hline
\end{tabular}

TABLE 1. (b). Journal of observations and photometry of Gl 229B.

\begin{tabular}{|c|c|c|c|c|c|c|c|}
\hline $\begin{array}{c}\text { Date } \\
95 \text { UT } \\
\text { (1) }\end{array}$ & $\begin{array}{l}\text { Bancl } \\
\text { (2) }\end{array}$ & $\begin{array}{c}\lambda \text { range } \\
(\mu \mathrm{mn}) \\
(3)\end{array}$ & $\begin{array}{c}\text { Telescope } \\
\text { (4) }\end{array}$ & $\begin{array}{l}\text { Instrument } \\
\text { (5) }\end{array}$ & $\begin{array}{l}\text { Mode } \\
\text { (6) }\end{array}$ & $\begin{array}{l}\text { mag } \\
\text { (7) }\end{array}$ & $\begin{array}{c}\mathbf{f}_{w} \\
(\mathrm{mJJy}) \\
(8)\end{array}$ \\
\hline 14 SEP & $\mathrm{Z}$ & $0.95-1.11$ & H200 & D.78 & Stare & 15.3 & 1.5 \\
\hline $14 \mathrm{SEP}$ & $\mathrm{J}$ & $1.15-1.40$ & $\mathrm{H} 200$ & D-78 & Stare & 14.2 & 3.2 \\
\hline 14 SEP & H & $1.49-1.81$ & II 200 & D-78 & Stare & 14.3 & 2.0 \\
\hline $14 \mathrm{SEP}$ & $\mathrm{Ks}$ & $1.90-2.32$ & $\mathrm{H} 200$ & D-78 & Stare & 14.3 & 1.3 \\
\hline $14 \mathrm{SEP}$ & $\kappa$ & $1.9-2.4$ & $\mathrm{H} 200$ & D-78 & Stare & 14.4 & 1.2 \\
\hline $23 \mathrm{NOV}$ & $\mathrm{K}$ & $2.02-\mathbf{2 . 4 3}$ & $\mathrm{H} 200$ & Pliotoneter (InSb) & Chop & 14.5 & 1.0 \\
\hline 6 OCT & 3.08 & $3.03-3.31$ & KECK & NIRC & Stare & 13.5 & $1.5 \pm 0.2$ \\
\hline $6 \mathrm{OCT}$ & 3.31 & $3.28-3.34$ & KECK & NIRC & Stare & $>14.4$ & $<0.6$ \\
\hline 6 OCT & 3.41 & $3.38-3.45$ & KECK & NIRC & Stare & $>14.7$ & $<0.4$ \\
\hline $23 \mathrm{NOV}$ & $L^{\prime}$ & $3.37-4.01$ & $\mathrm{H} 200$ & Photometer(InSb) & Chop & 13.4 & $1.1 \pm 0.8$ \\
\hline 11 OCT & 3.82 & $3.67-3.97$ & $\mathrm{H} 200$ & D. 78 & Chop & 11.8 & $5.0 \pm 1.0$ \\
\hline $6 \mathrm{OCT}$ & 3.99 & $3.97-4.02$ & KECK & NIRC & Stare & 11.4 & $6.6 \pm 1.0$ \\
\hline $23 \mathrm{NOV}$ & $\mathrm{M}$ & $4.47-5.08$ & H200 & Photoneter(InSh) & Chop & 10.9 & $7 \pm 3$ \\
\hline $9 \& 22$ NOV & $\mathrm{N}$ & $8.0-13.1$ & Hโ200 & Bolometer $(\mathrm{Ge})$ & Chop & 9.9 & $4.2 \pm 0.9$ \\
\hline
\end{tabular}

Col. (4): $\mathrm{H} 200=$ the Hale 200 -inch telescope.

Col. (5): Both D-78 and NIRC employ SBRC InSl, $250 \times 256$ arrays.

Col. (8): $1 \sigma$ error in flux density is estinated to be $\pm 10 \%$, urless given explicitly.

sky buffers from the sum of the two image buffers.

Gl 229B was observed five times at 1.8 airmass by this procedure while HD 22686 (Elias et al. 1982) was observed twice at 1.7 airmass for 1 instead of 100 cycles.

\subsection{Narrowband Imaging at the Keck Telescope}

Gl 229B was imaged through narrowband filters in the 3 $\mu \mathrm{m}$ atmospheric window in late twilight and morning daylight of 1995 October 6 UT at the Keck Telescope using NIRC (Matthews \& Soifer 1994). The sky was photometric and the seeing at $3 \mu \mathrm{m}$ was about 0.5 arcsec. The observations were unguided but of short enough duration that no tracking errors were noticed in the resulting images. The object images were obtained by summing a series of short integrations into a buffer and then the telescope was offset by 15 arcsec north and a sky frame was obtained in the same manner. The standard star HD 22686 was measured, and then Gl 299B was measured. Both were at similar airmass. In no case, did any of the six diffraction spikes produced by the secondary supports and segment gaps interfere with the image of Gl 229B.

\subsection{Observations by Aperture Photometer}

Observations at $N$ band were made on 1995 November 9 and 22 (UT) at the $f / 70$, infrared Cassegrain focus of the Hale 200-inch telescope with a Germanium bolometer (Infrared Laboratories, Tucson, AZ). The observations at the $K$, $L^{\prime}$, and $M$ bands were obtained on 1995 November 23 UT using a photovoltaic InSb detector (SBRC, Santa Barbara, CA). Table 2 shows the details of the observations. The visual seeing estimated from the images displayed on the TV acquisition camera were 1.5 arcsec for the $N$ band observations and 1 arcsec for the $K, L^{\prime}$, and $M$ observations. Experience has shown that the seeing diameter at $N$ band is about one half of that at visual wavelength on the Hale 200-inch telescope. The sky was photometric during all of the observations.
For all observations the focal plane aperture was 2.0 arcsec in diameter and the chopping was east-west with a square wave of $50 \mathrm{~Hz}$ for the bolometer and $5 \mathrm{~Hz}$ for the InSb detector. The square wave had a peak-to-peak amplitude of 4.8 arcsec. This amplitude was chosen to be twice the right ascension distance from Gl 229B to the secondary support diffraction spike from Gl 229A. This configuration effectively nulled the contribution of the diffraction spike to the signal of Gl 229B when chopping across the spike. The signal was demodulated using a lock-in amplifier using the chopper signal as reference. The output of the lock-in amplifier was converted to pulses by a voltage-to-frequency converter and integrated by a digital counter. A time of $\tau$ seconds of data were taken at one telescope position, after which the beams were switched by moving the telescope east or west by 4.8 arcsec and another $\tau$ seconds of data were taken. The difference between these two integrations constitutes a measure of the signal, called a pair. The east-west order was then reversed and the process was repeated so that an even number $(P)$ of pairs was obtained in all cases. See Table 2 for values of $P$ and $\tau$.

During all observations, a TV autoguider on an offset guider stage was used to obtain and preserve centering of the objects in the focal plane aperture. The offset guider has an astrometric uncertainty of less than 0.1 arcsec peak amplitude when used to offset between objects. The guider also corrected for the change in the differential refraction of the atmosphere between the guide star at $0.7 \mu \mathrm{m}$ and the infrared source. For Gl 229B observations we used a guide star located 19 arcsec east and 70 arcsec north from G1 229A.

The above is standard procedure for observations with an infrared photometer. The fast chopping and beam switching

TABLE 2. Details of aperture photometry.

\begin{tabular}{|c|c|c|c|c|c|c|c|}
\hline $\begin{array}{c}\text { Date } \\
\text { (05 UT) }\end{array}$ & Band & Object & $\begin{array}{c}\tau \\
(\mathrm{sec})\end{array}$ & $\underset{\text { (pair) }}{\mathbf{P}}$ & $\begin{array}{l}T_{\text {int }} \\
(\mathrm{sec})\end{array}$ & Airmass & $\begin{array}{c}\text { Signal } \\
\text { (DN/sec) }\end{array}$ \\
\hline \multirow[t]{10}{*}{$9 \mathrm{NOV}$} & $\mathrm{N}$ & HR 2491 & 20 & 1 & 20 & 1.9 & $2.37 \times 10^{6}$ \\
\hline & & Gl $229 \mathrm{~A}$ & 20 & 4 & 80 & 1.9 & $1.81 \times 10^{4}$ \\
\hline & & G1 $229 \mathrm{~B}$ & 40 & 64 & 2560 & 1.8 & $26 \pm 31$ \\
\hline & & Gl $220 \mathrm{~A}$ & 20 & 4 & 80 & 1.8 & $2.02 \times 10^{4}$ \\
\hline & & Gl $229 \mathrm{~B}$ & 40 & 64 & 2560 & 1.8 & $58 \pm 31$ \\
\hline & & Gl 220I & 40 & 64 & 2560 & 1.9 & $-4.5 \pm 31$ \\
\hline & & Gl $229 \mathrm{~A}$ & 20 & 2 & 40 & 1.9 & $1.83 \times 10^{4}$ \\
\hline & & Gl 2291 & 40 & 64 & 2560 & 2.2 & $28.5 \pm 31$ \\
\hline & & Gl 229A & 20 & 2 & 40 & 2.4 & $1.77 \times 10^{4}$ \\
\hline & & HRR 2491 & 20 & 2 & 40 & 1.9 & $2.34 \times 10^{6}$ \\
\hline \multirow[t]{9}{*}{$22 \mathrm{NOV}$} & $\mathbf{N}$ & HR 2491 & 20 & 4 & 80 & 2.1 & $2.78 \times 10^{6}$ \\
\hline & & (i) $229 \mathrm{~A}$ & 20 & 4 & 80 & 2.0 & $2.03 \times 10^{4}$ \\
\hline & & Gl $229 \mathrm{~B}$ & 40 & 64 & 2560 & 1.8 & $111 \pm 34$ \\
\hline & & Gl $229 \mathrm{~A}$ & 20 & 6 & 120 & 1.8 & $1.90 \times 10^{4}$ \\
\hline & & Gl $220 \mathrm{I}$ & 40 & 64 & 2560 & 1.8 & $2.4 \pm 34$ \\
\hline & & G) $229 \mathrm{~A}$ & 20 & 4 & 80 & 1.9 & $1.62 \times 10^{4}$ \\
\hline & & G1 $229 \mathrm{~B}$ & 40 & 64 & 2560 & 2.0 & $113 \pm 34$ \\
\hline & & (G) $229 \mathrm{~A}$ & 20 & 4 & 80 & 2.2 & $1.46 \times 10^{4}$ \\
\hline & & HR 2491 & 20 & 4 & 80 & 1.8 & $2.11 \times 10^{6}$ \\
\hline \multirow[t]{11}{*}{23 NOV } & $\mathbf{M}$ & HR 2491 & 20 & 2 & 40 & 2.2 & $1.89 \times 10^{6}$ \\
\hline & $\mathbf{L}^{\prime}$ & HR 2401 & 20 & 2 & 40 & 2.2 & $6.24 \times 10^{7}$ \\
\hline & $L^{\prime}$ & Gl $229 \mathrm{~A}$ & 20 & 2 & 40 & 2.0 & $4.49 \times 10^{5}$ \\
\hline & $M$ & (i) $229 \mathrm{~A}$ & 20 & 2 & 40 & 2.0 & $1.37 \times 10^{4}$ \\
\hline & $\mathbf{K}$ & Gi $229 \mathrm{~A}$ & 20 & 2 & 40 & 2.0 & $3.95 \times 10^{7}$ \\
\hline & K & (il $229 \mathrm{~B}$ & 80 & 2 & 160 & 1.9 & $4.35 \times 10^{3}$ \\
\hline & $\mathrm{K}$ & (d) $220 \mathrm{I}$ & 80 & 2 & 160 & 1.9 & $2.03 \times 10^{3}$ \\
\hline & $\mathrm{K}$ & Gi $229 \mathrm{~B}$ & 40 & 2 & 80 & 1.9 & $5.23 \times 10^{3}$ \\
\hline & M & G1 229B & 80 & 32 & 2560 & 1.9 & $28.5 \pm 20$ \\
\hline & M & Gl $220 \mathrm{~A}$ & 20 & 2 & 40 & 1.8 & $1.40 \times 10^{4}$ \\
\hline & $\mathrm{K}$ & Gl $229 \mathrm{~B}$ & 20 & 2 & 40 & 1.8 & $4.68 \times 10^{3}$ \\
\hline & M & Gl $229 \mathrm{~B}$ & 40 & 64 & 2560 & 1.8 & $6.8 \pm 20$ \\
\hline & $\mathrm{M}$ & (GI $220 \mathrm{~A}$ & 20 & 2 & 40 & 1.8 & $1.48 \times 10^{4}$ \\
\hline & $\mathbf{K}$ & Gl $229 \mathrm{~B}$ & 20 & 2 & 40 & 1.8 & $4.87 \times 10^{3}$ \\
\hline & M & (11 $229 \mathrm{~B}$ & 40 & 64 & 2560 & 1.8 & $31.0 \pm 20$ \\
\hline & M & Gi $229 \mathrm{~A}$ & 20 & 2 & 40 & 1.9 & $1.31 \times 10^{4}$ \\
\hline & $\mathbf{L}^{\prime}$ & G1 $229 \mathrm{~A}$ & 20 & 2 & 40 & 1.9 & $4.36 \times 10^{4}$ \\
\hline & L' & G1 $229 \mathrm{~B}$ & 80 & 8 & 640 & 1.9 & $79 \pm 53$ \\
\hline & $\mathbf{K}$ & Cl $229 \mathrm{~B}$ & 20 & 2 & 40 & 2.0 & $4.30 \times 10^{3}$ \\
\hline & $\mathbf{M}$ & Gl 2203 & 40 & 64 & 2500 & 2.1 & $28.8 \pm 20$ \\
\hline & $\mathbf{M}$ & Gl $229 \mathrm{~A}$ & 40 & 2 & 80 & 2.3 & $1.06 \times 10^{4}$ \\
\hline & $M$ & HR 2401 & 20 & 2 & 40 & 1.8 & $1.99 \times 10^{6}$ \\
\hline
\end{tabular}


removes slowly varying sky and instrument contributions. In addition, in order to measure the possible contribution of scattered light from G1 229A to Gl 229B we performed a series of observations centered on a position inverted about Gl 229A. This point, hereafter Gl 229I, is located at 2.4 arcsec west and 7.3 arcsec north of Gl 229A. For Gl 229I, we beam switched in a manner identical to that used for Gl 229B.

In Table 2 we purposely present the raw data (with no corrections applied) to demonstrate the stability and consistency of the observations which were made with an usually small focal plane aperture and near a very bright source, Gl 229A. In Table 2, for those measurements with no listed uncertainties, the dominant cause of variation is due to fluctuations in the seeing; for those cases, the uncertainty is estimated to be $\pm 10 \%$. The total range in airmass correction is a maximum of $\pm 5 \%$ in the $M$ band and is about one half that in the other bands for both the standard (HR 2491, see below) and Gl 229A. However, the airmass correction requires an accurate knowledge of the spectrum of the object. The spectrum of Gl 229B is quite complicated (see Sec. 3). For simplicity, we assume an airmass correction for Gl 229B to be the same as that of the other stars.

The reduced photometric data are shown in Table 1. The uncertainties where shown are derived from the nightly average of the statistical fluctuations within each individual measurement. There is no evidence that the noise, exclusive of seeing variations, varied over a night's measurements.

The standard star HR 2491 (Sirius; Neugebauer 1995) was measured both immediately before and after each night's observations of the Gl 229 system. The observations were conducted in a manner identical to that used for Gl 229 including the use of an offset guide star. HR 2491 was too bright to measure in the $K$ band, so its measurement at $L^{\prime}$ plus the known stable ratio of system response to flux in the $K$ band to that in the $L^{\prime}$ band was used to derive the $K$ band magnitudes of Gl 229A and Gl 229B. In the case of the $K$ band measurement of Gl 229B only, the data in Table 2 are derived from the flux measured at the position of Gl 229B with the flux measured at the Gl 229I position subtracted; this reduces the raw value by about one half. It should be noted that discrepancy of this $K$ result from that reported in N95 led us to reanalyze the imaging data reported in N95.

The ratio of the signal at Gl 229I to Gl 229A forms a good estimate of the contribution of the scattered light from Gl 229A to the signal measured at Gl 229B. Seeing improves and the strength of the diffraction spikes decreases with increasing wavelength. Thus, the scattered light contributions are expected to decrease with increasing wavelength. An upper bound to the scattered light, at longer wavelengths, is given by the measured signal of Gl 229A multiplied by the ratio of the $K$ signal of Gl 229I to Gl 229A. The total error is due to both this scattered light and also measurement errors. The ratios of the expected scattered light contribution to the measurement error are 0.43 at $L^{\prime}, 0.07$ at $M$, and 0.06 at $N$. For this reason, no attempt was made to measure Gl 229I at $M$, and the measurement at $N$ of Gl 229I, $0.5 \pm 1.0 \mathrm{mJy}$, was not included in the final photometry of Gl 229B. The measurement at $L^{\prime}$ is clearly uncertain. It is included as a check

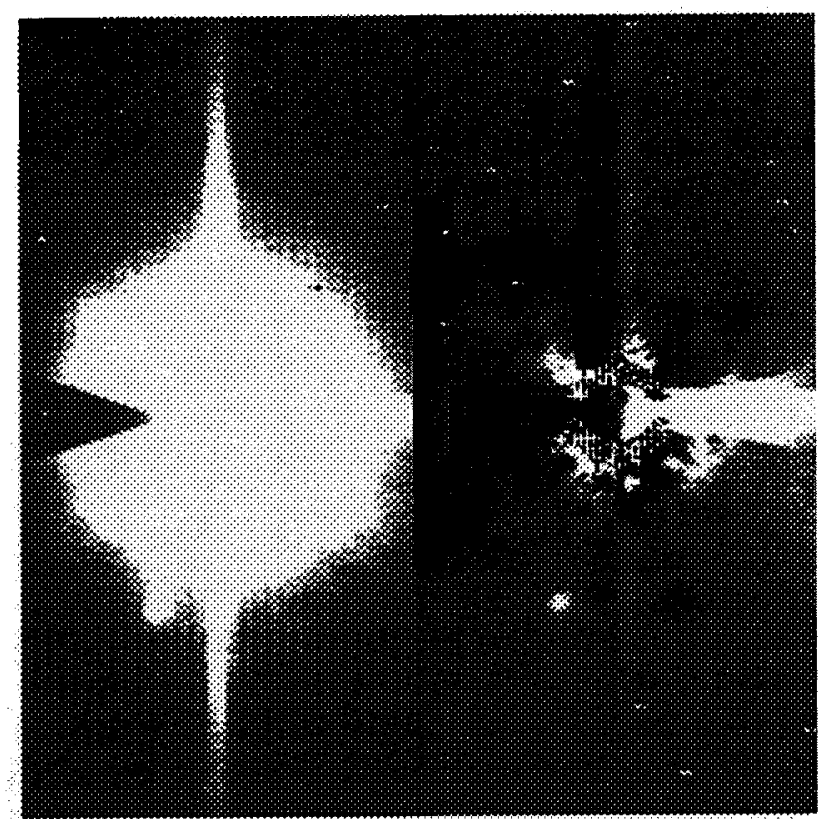

FIG. 1. (Left) The $K_{s}$ image used in N95. (Right) The difference between the left image and its reflection along the vertical line. The stretch is \pm 150 ADU. Note how flat the sky is, especially in the vicinity of Gliese $229 \mathrm{~B}$. Most photometry packages do not work well when the sky is not reasonably flat. We used the image on the right side to derive the photometry in the near-IR bands.

on the narrow band measurements and shows that there is no truly bright emission feature between these bands.

\section{REDUCTION OF IMAGING AND SPECTROSCOPIC DATA}

\subsection{Revision of the Near-infrared Magnitudes}

In the lower right panel of Fig. 1 of N95, a $K \mathrm{~s}(2.15 \mu \mathrm{m})$ image of Gl 229B is shown. At this wavelength, the brightness ratio of Gl $229 \mathrm{~A}$ to $\mathrm{Gl} 229 \mathrm{~B}$ is $\approx 10^{4}$. In the case of infrared imaging, Gl 229A is occulted by a focal plane disk, but there is no special Lyot stop to suppress diffracted light from the telescope edges and secondary mirror supports as with the coronagraph used at optical wavelengths. In the initial reduction whose photometric results were reported in N95, DAOPHOT in IRAF was used to fit the point spread function of Gl 229B in the presence of the wing of the point spread function of G1 229A. The large discrepancy between the $K$ magnitude in $\mathrm{N} 95$ and that obtained by the aperture photometry described in the previous section, led us to reanalyze all the near-infrared imaging data. In this reanalysis, we produced a flattened image by subtracting the image reflected about the vertical diffraction spike from the original image. As can be seen from Fig. 1, the difference between this flattened image and the image used in N95 is striking. The new image is sufficiently flat so that accurate photometry can be obtained using APPHOT in IRAF. The revised magnitudes for the $Z, J, H, K \mathrm{~s}$, and $K$ are given in Table 1 .

\subsection{3 um Narrowband Data Reduction}

Narrowband images at $3.08,3.31,3.41$, and $3.99 \mu \mathrm{m}$ obtained at the Keck Telescope were reduced differently from the broadband images obtained at the Hale 200-inch tele- 


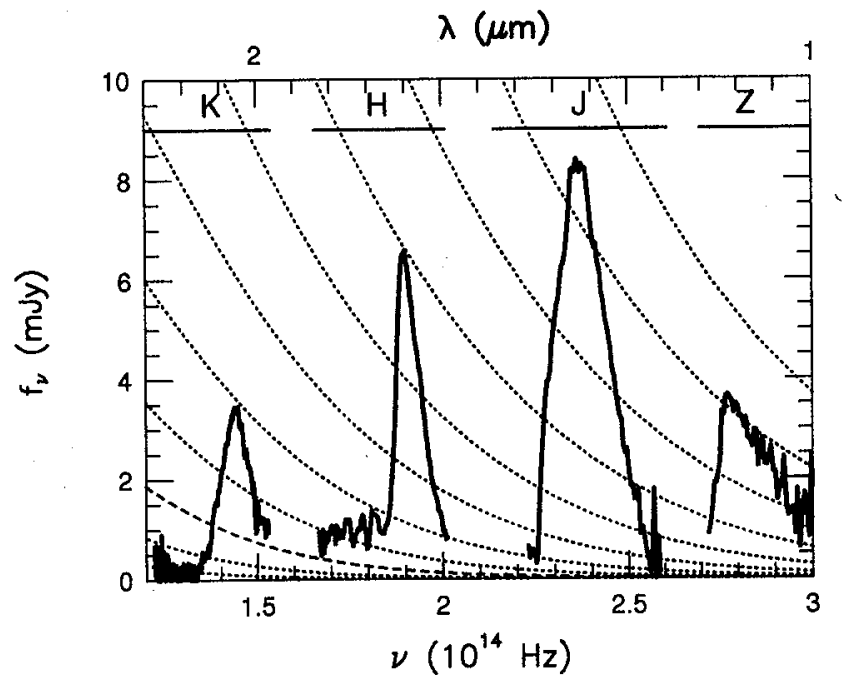

FyG. 2. The observed near-infrared spectra of Gl 229B (solid lines). The spectral resolution is $\nu / \Delta \nu \approx 150$. The width of each window in the Earth's atmosphere is indicated by the horizontal line just below the name of the window. Dashed lines indicate blackbody curves for $T=700 \mathrm{~K}$ (lower left) to $1700 \mathrm{~K}$ (upper right) plotted at an interval of $100 \mathrm{~K}$. The long-dashed line is for $T=900 \mathrm{~K}$, the estimated value of $T_{\text {eff }}$ for Gl 229B. Each blackbody curve was calculated assuming the radius, $R=0.1 R_{\odot}$, and the distance, $d=5.7 \mathrm{pc}$, which are the assumed parameters for Gl $229 \mathrm{~B}$. Each intersection of the spectrum and a blackbody curve indicates the brightness temperature $\left(T_{B}\right)$ of that point of the spectrum. The highest, $T_{B}=1640 \mathrm{~K}$, corresponds to the $J$ band peak where the deepest layer in the atmosphere is exposed, while the lowest, $T_{B}=700 \mathrm{~K}$, corresponds to the bottom of the methane absorption in $K$ band where only the upper layer in the atmosphere is seen.

scope. Because of the excellent seeing and low scattered light in the $3 \mu \mathrm{m}$ region at Mauna Kea, the point spread function of Gl 229B was hardly affected by the wing of Gl 229A. However, vertical stripes were present in each image due to a readout electronics problem. This artifact was removed by shifting the image vertically and then subtracting the shifted image from the original. Gl 229B was detected at 3.08 and $3.99 \mu \mathrm{m}$, but was not detected at 3.31 and $3.41 \mu \mathrm{m}$. For 3.31 and $3.41 \mu \mathrm{m}, 1 \sigma$ upper limits of the fluxes are given in Table 1.

\subsection{Reduction of the Near-infrared Grism Spectra}

Along with the imaging data obtained 1995 September 14 UT, we have reanalyzed the two-dimensional grism spectra obtained on 1995 September 14 and 15 UT. The observational procedures are briefly described in $\mathrm{O} 95$. Here we elaborate on the rereduction and racalibration of the spectra. In the spectral images, the spatial (slit) direction was placed along the $y$ axis and the dispersion direction was along the $x$ axis. In the original spectral image, the pixel-wise dispersion $(\Delta \lambda /$ pixel) is a weak function of $y$. Using the strong emission lines seen in the spectrum of the bright planetary nebula, NGC 7027, this gradient of pixel-wise dispersion was estimated and each spectral image was rectified so that the pixelwise dispersion became uniform along the $y$ axis. This procedure was performed before the column-wise subtraction of the $\mathrm{OH}$ airglow lines by the routine, BACKGROUND, in IRAF. The first-order blaze wavelength of the grism is $6.6 \mu \mathrm{m}$, and the orders in individual bands are third in $K$, fourth in $H$,
TABLE 3. Absolute magnitudes and In-band luminosities of GL 229B

\begin{tabular}{cccc}
\hline \hline Band & Absolute Mag & $\begin{array}{c}\mathrm{f}_{\nu}(\mathrm{d}=10 \mathrm{pc}) \\
(\mathrm{mJy})\end{array}$ & $\begin{array}{c}\text { Luminosity } \\
\left(\mathrm{L}_{\odot}\right)\end{array}$ \\
\hline Gunn r & $>23.3$ & $<0.0014$ & $<3.0 \times 10^{-9}$ \\
Gunn i & 21.2 & $0.0084 \pm 0.002$ & $2.2 \times 10^{-8}$ \\
Gunn z & 19.0 & $0.061 \pm 0.02$ & $1.0 \times 10^{-7}$ \\
$Z$ & 16.5 & 0.50 & $6.9 \times 10^{-7}$ \\
$\mathrm{~J}$ & 15.4 & 1.1 & $1.5 \times 10^{-8}$ \\
$\mathrm{H}$ & 15.5 & 0.68 & $7.2 \times 10^{-7}$ \\
$\mathrm{Ks}$ & 15.5 & 0.44 & $2.9 \times 10^{-7}$ \\
$\mathrm{~K}$ & 15.6 & 0.38 & $2.8 \times 10^{-8}$ \\
3.08 & 14.7 & $0.5 \pm 0.1$ & $5.8 \times 10^{-9}$ \\
3.31 & $>15.6$ & $<0.19$ & $<1 \times 10^{-8}$ \\
3.41 & $>15.9$ & $<0.14$ & $<8 \times 10^{-8}$ \\
$\mathrm{~L}$ & 14.6 & $0.4 \pm 0.3$ & $\mathbf{1 . 6 \times 1 0 ^ { - 7 }}$ \\
3.82 & 13.0 & $1.6 \pm 0.3$ & $3.1 \times 10^{-7}$ \\
3.99 & 12.6 & $2.2 \pm \mathbf{0 . 3}$ & $6.3 \times 10^{-8}$ \\
$\mathrm{M}$ & 12.1 & $2.2 \pm 1.0$ & $6.1 \times 10^{-7}$ \\
$\mathrm{~N}$ & 11.1 & $1.4 \pm \mathbf{0 . 3}$ & $5.9 \times 10^{-7}$ \\
\hline
\end{tabular}

Lines 1,2 , and 3: Data from N05.

Col 3: Flux density if Gl $229 \mathrm{~B}$ were placed at $10 \mathrm{pc}$.

fifth and sixth in $J$, seventh and eighth in $Z$. The $Z$ band spectra are thus obtained at angles well off the blaze angle. Consequently, the efficiency in the $Z$ band is quite low. After eliminating bad pixels, one-dimensional spectra were extracted. For the calibration of atmospheric transmission, HR $1747\left(\mathrm{G} 0 \mathrm{~V}, T_{\text {eff }}=5900 \mathrm{~K}\right)$ and HR $863\left(\mathrm{G} 5 \mathrm{~V}, T_{\text {eff }}=5660\right.$ $\mathrm{K})$ were used. $\mathrm{G}$ dwarfs are known not to have appreciable absorption or emission features at a resolution of about 150 in the near infrared. In the flux calibration, each raw spectrum divided by one of the two $G$ star spectra was multiplied by the blackbody spectrum with corresponding $T_{\text {eff }}$ normalized by the photometric data given in Table 1 . The resultant near-infrared spectra of Gl 229B are given in Fig. 2. The same spectra in a numerical form (ASCII file) can be obtained through the web page (http://astro. caltech.edu/ srk). The revised spectra differ from those in 095 mainly in the flux calibration.

\section{DISCUSSION}

\subsection{Photospheric Emission of Gl 229B}

The near-infrared spectrum of Gl 229B, presented in Fig. 2, can be characterized by deep absorption features of $\mathrm{H}_{2} \mathrm{O}$ seen in the low-frequency side of $Z$, both sides of $J$, and the high-frequency halves of $H$ and $K$. The other dominant features are absorption by $\mathrm{CH}_{4}$ seen in the low-frequency halves of $H$ and $K$. The fact that strong $\mathrm{H}_{2} \mathrm{O}$ absorption features dominate the transmission by the Earth's atmosphere at these wavelengths must be accounted for in the determination of the total flux from Gl 229B. In the Earth's cool atmosphere, only low-order rotational states of $\mathrm{H}_{2} \mathrm{O}$ are excited, while in the warmer atmosphere of $\mathrm{Gl} 229 \mathrm{~B}$, higher-order rotational states are also excited. Higher excitation of $\mathrm{H}_{2} \mathrm{O}$ in the atmosphere of $\mathrm{Gl} 229 \mathrm{~B}$ results in much wider absorption bands. Thus it is expected that most of the emission from Gl 229B will be observed by ground-based broadband photometry. In Table 3, we present, for each band, the absolute magnitude, corresponding flux in mJy if Gl 229B were at the distance of $10 \mathrm{pc}$, and $L_{\nu}$, the luminosity measured within the filter bandwidths. Here $L_{\nu} \equiv f_{\nu} 4 \pi d^{2} \Delta \nu$; where $f_{\nu}$ is the flux density at frequency $\nu, d$ is the distance to the source, $5.7 \mathrm{pc}$; and $\Delta \nu$ is the bandwidth of the band as given in Table 1(B). 
The total in-band observed luminosity $\left(L_{\mathrm{obs}}\right)$ is $(4.9 \pm 0.6) \times 10^{-6} L_{\odot}$. The error estimate includes the error in G1 229A's parallax and uncertainties in the observations. If we assume that the radius of $\mathrm{Gl} 229 \mathrm{~B}$ [hereafter $\mathrm{R}(\mathrm{Gl} 229 \mathrm{~B})$ ] is $0.1 R_{\odot} \approx R_{J}$, then $T_{\text {eff }}>856 \mathrm{~K}$.

\subsection{Comparison with Models and Bolometric Correction}

We compare the atmospheric models of Tsuji et al. (1995, 1996) with our data. Their models cover the wavelength range between 0.2 and $45 \mu \mathrm{m}$ and their model for $T_{\text {eff }}=1000$ $K$ reproduces the observed spectral energy distribution (SED) reasonably well (Tsuji et al. 1996). Tsuji has kindly provided us with his model spectra for $T_{\text {eff }}=800,900,1000$, and $1200 \mathrm{~K}$. These models give the following ratios of the luminosity that is contained in our observed bands to the bolometric luminosity $\left(L_{\text {obs }} / L_{\text {bol }}\right): 0.76,0.77,0.78$, and 0.80 . Therefore $L_{\mathrm{obs}} / L_{\mathrm{bol}}$ is rather insensitive to the effective temperature of the model. Using $L_{\mathrm{obs}} / L_{\mathrm{bol}}=0.77$ for a bolometric correction, we obtain our best estimate of $L_{\text {bol }}=6.4 \times 10^{-6}$ $L_{\odot}$. Again assuming $R(\mathrm{Gl} 229 \mathrm{~B})=0.1 \quad R_{\odot}$, we obtain $T_{\text {eff }}=913 \mathrm{~K}$. The observed fluxes are compared with the model spectrum for $T_{\text {eff }}=900 \mathrm{~K}$ in Fig. 3 in which overall agreement is obvious.

\subsection{Brightness Temperature and Depths of the Observed Atmosphere}

The SED of Gl 229B is significantly different from that of the blackbody for $T_{\text {eff }}=900 \mathrm{~K}$. Due to deep $\mathrm{H}_{2} \mathrm{O}$ and $\mathrm{CH}_{4}$ absorption bands, the emergent flux is squeezed into the narrow windows through which hotter regions in the lower atmosphere are exposed. Figure 2 presents the observed nearinfrared spectrum with blackbody curves superimposed. Each intersection of the spectrum and a blackbody curve indicates the brightness temperature $\left(T_{B}\right)$ of that portion of the spectrum. The highest, $T_{B}=1640 \mathrm{~K}$, is seen at the peak within the $J$ band spectrum $\left(\nu=2.36 \times 10^{14} \mathrm{~Hz}\right)$. Figure 3 shows the observed SED and the model spectrum with the blackbody curves similarly superimposed. Nondetections with the narrowband filters at 3.31 and $3.41 \mu \mathrm{m}[\log (\nu)$

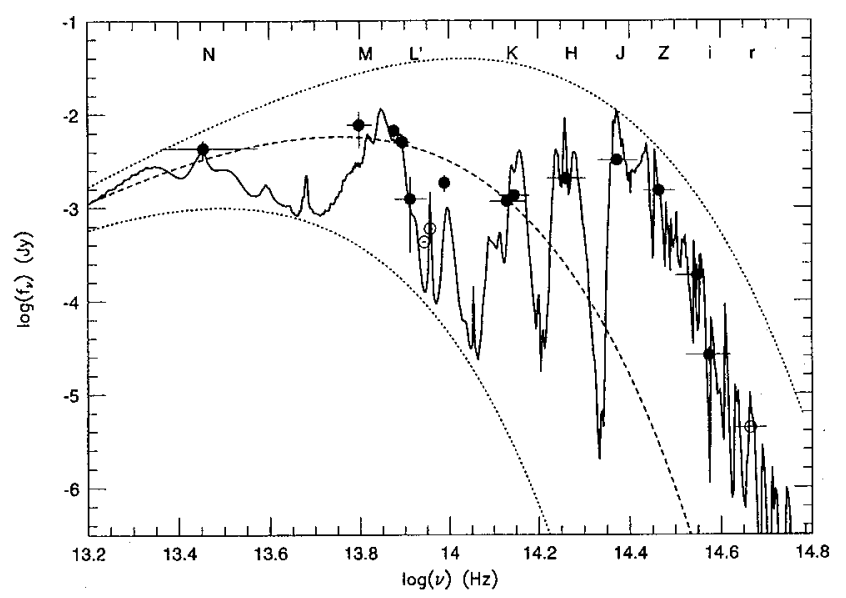

FIG. 3. Comparison of broadband photometry with the model spectrum. Filled circles denote detections, while open circles indicate $1 \sigma$ upper limits. Horizontal bars attached to circles denote bandwidths, while vertical bars indicate $1 \sigma$ photometric errors. Locations of major bands are indicated by the letters. The solid line is the model spectrum for $T_{\text {eff }}=900 \mathrm{~K}$ by Tsuji et al. Two short-dashed lines indicate blackbody curves for $T=500 \mathrm{~K}$ (bottom) and $1700 \mathrm{~K}$ (top). The long-dashed line indicates a blackbody curve for $T=900 \mathrm{~K}$, the estimated value of $T_{\text {eff }}$ for GI $229 \mathrm{~B}$. Nondetections through the narrowband filters at 3.31 and $3.41 \mu \mathrm{m}[\log (\nu) \approx 13.95]$ indicate that the upper most methane layer is cooler than $600 \mathrm{~K}$. For the model spectrum and the blackbody spectra, $R=0.1 R_{\odot}$ and $d=5.7 \mathrm{pc}$ are assumed.

$\approx 13.95$ ] indicate that the upper most methane layer is cooler than $600 \mathrm{~K}$.

We thank T. Tsuji for providing us with his model spectra and for comments on the manuscript and P. Goldreich and P. Nicholson for helpful discussion. A part of the observations was obtained at the W. M. Keck Observatory on Mauna Kea, Hawaii, which is operated jointly by the California Institute of Technology and the University of California. We thank P. Nicholson and C. Lawrence for providing some of the Hale 200-inch telescope time and B. T. Soifer for the Keck Telescope time for these observations. Much of the research activity reported here is supported by the Packard Foundation, NSF, and NASA. B.R.O. is supported by an NSF Graduate Research Fellowship. Infrared astronomy at Palomar was supported by a grant from the NSF.

\section{REFERENCES}

Elias, J. H., Frogel, J. A., Matthews, K., \& Neugebauer, G. 1982, AJ, 87, 1029

Matthews, K., \& Soifer, B. T. 1994, in Infrared Astronomy with Arrays: The Next Generation, edited by S. I. McLean (Kluwer, Dordrecht), p. 239

Nakajima, T., Oppenheimer, B. R., Kulkarni, S. R., Golimowski, D. A., Matthews, K., \& Durrance, S. T. 1995, Nature, 378, 463 (N95)
Neugebauer, G. 1995 (personal communications)

Oppenheimer, B. R., Kulkarni, S. R., Matthews, K., \& Nakajima, T. 1995, Science, 270, 1478 (O95)

Tsuji, T., Ohnaka, K., \& Aoki, W. 1995, in The Bottom of the Main Sequence--and Beyond, edited by C. G. Tinney (Springer, Berlin)

Tsuji, T., Ohnaka, K., Aoki, W., \& Nakajima, T. 1996, A\&AL (submitted) 
